\title{
The Neuroprotective Effect of Atorvastatin on Apoptosis of Hippocampus Following Transient Global Ischemia/ Reperfusion
}

\author{
Masoumeh Faghani ${ }^{1}$, Farhang Ejlali ${ }^{1}$, Zahra Nadia Sharifi ${ }^{2}$, Hassan Molladoost ${ }^{3}$, Shabnam Movassaghi ${ }^{2 凶}$
}

\author{
${ }^{1}$ Anatomy Department, Guilan University of Medical Sciences, Rasht, Iran \\ ${ }^{2}$ Anatomical Sciences Department, Islamic Azad University, Tehran Medical Sciences Branch, Tehran Iran \\ 3 Physiology Department, Guilan University of Medical Sciences, Rasht, Iran
}

\begin{abstract}
Background: Hippocampus CA1 cells are highly sensitive to ischemia. Because of anti-oxidative property, atorvastatin by eliminating free radicals and other constituent due to cell lesions prevents damages or death of intact cells. The aim of this study is to determine neuroprotective effect of atorvastatin on apoptosis of hippocampus CA1 cells in male rats. Materials and Methods: In an experimental study 24 male rats -Wistar adult race- were divided into four groups: Ischemic, vehicle, treatment and control. In order to make an ischemic- reperfusion model, common carotid artery was clamped bilaterally for 20 minutes. In treatment group, the first dose of Atorvastatin $(10 \mathrm{mg} / \mathrm{kg})$ was injected six hours after ischemic- reperfusion and 24,48 and 72 later intraperitoneally. Four days after ischemic- reperfusion tissue section were obtained and stained using Nissl method. Then intact healthy pyramidal CA1 hippocampus cells were counted. TUKEY and One Way ANOVA were used for analyzing the obtained results. Results: No significant difference was found in numbers of healthy intake pyramidal and apoptotic cells of CA1 hippocampus region between treatment group $(10 \mathrm{mg} / \mathrm{kg}$ Atorvastatin) and controls, whereas a significant reduction of these cells was observed in ischemia and vehicle groups in comparison to controls $(\mathrm{P}<0.05)$. Conclusion: Using $10 \mathrm{mg} / \mathrm{kg}$ atorvastatin following cerebral ischemia- reperfusion has a protective effect on pyramidal cells of CA1 region in hippocampus in male rats leading to reduction of degeneration and apoptosis.[GMJ.2016;5(2):82-89]
\end{abstract}

Keywords: Atorvastatin; Hippocampus; Ischemia; Neuroprotective

\section{Introduction}

$\mathrm{C}$ erebral ischemia is a well-known major common problem worldwide. Stroke is the most important cause of cerebral ischemia and the third cause of death after heart infarction and cancer in western countries. Cerebral ischemia will lead to several disorders such as motor, sensory, visual complication,

\section{GMJ}

o2016 Galen Medical Journal

Fax: +98 7312227091

PO Box 7461686688

Email:info@gmj.ir aphasia and behavioral disorders especially spatial learning disorders [1-3]. Reperfusion lesions are those complications caused by establishment of blood stream in tissue after an ischemia period. Lack of oxygen and other nutrients leads to a situation in which re-establishment of blood stream, instead of natural tissue activity, produces inflammation and oxidative lesions by inducing oxidative

\footnotetext{
Correspondence to:

Shabnam Movassaghi, Faculty of Medicine, Islamic

Azad University, Tehran Medical Sciences Branch,

Tehran, Iran

Telephone Number: +9821-22006660

Email Address: shmovasaghi@iautmu.ac.ir
} 
stress [4]. Investigators have shown that common carotid bilateral closure for a few minutes causes ischemia in hippocampus, CA1 neurons and hilus are the most susceptible region for damage [5]. Regarding to vital role of hippocampus, reducing damage and repairing this region following diseases and traumatic lesion are of great importance [6].

Hippocampus is located in the bottom of lateral ventricle in temporal lobe. This area plays a role in formation of new memory and spatial information processing and is among the first areas influenced by degenerative ischemic diseases and traumatic damages [7, 8]. Hippocampus is very sensitive to hypoxia and traumatic damages and numerous functional disorders will emerge after ischemic- reperfusion damages [9]. Upon ischemic- reperfusion, free radicals of oxygen and other oxidants will be formed which attack target tissues and cause cell destruction more than ischemia alone [10]. During generalized cerebral ischemia in rats, specific neural destruction occurs inhippocampusCA1 region and dorsolateral of striatum mostly in striatum in first 24 hours and in hippocampus after 72 hours. As a result, neurons in hippocampus have a longer time for survival than other parts of brain. Pulsienelli et al called it delayed cell death which yields a proper opportunity for treatment and prevention of cell death [11].

Thrombolysis is used for eliminating atherosclerotic lesions which cause ischemia [12]. Atorvastatin- a member of statins- is used nowadays as an important drug for cerebral ischemic lesions. Statins inhibit the process of changing Malonate to HMG-COA and thus reduce cholesterol production [13]. Atorvastatin is capable of increasing cortical neurons resistance to cell death and oxidative damages due to ischemia [14]. Prolonged administration and preventive treatment using statins induces reduction of stroke extent and improvement of neural damages in rats with cerebral ischemia [15]. Moreover, atorvastatin has a protective effect in periods pre and post-ischemia [16] and more lymphophilic impact and longer half-life in inhibiting HMG-COA Reductase enzyme [17].Besides reducing serum cholesterol, Atorvastatin has a wide range of anti-inflammatory and anti-oxidative proper- ties, and prevents atherosclerotic lesions [18] and regulates nitric oxide synthesis in endothelial cells which leads to elevation of capillary blood stream [19].

This drug protects brain in inflammatory damages and adjusts inflammatory factors playing on important role in inhibiting ischemic inflammatory damages [20]. Due to lack of investigation on the effect of atorvastatin after cerebral ischemia in hippocampus CA1 region, this study was conducted to recognize its effect on improvement of cerebral ischemia and neural cell death of hippocampus in Wistar rat following ischemia-reperfusion.

\section{Materials and Methods}

Twenty-four adult male Wistar rats weighting 250-300gr were obtained from animal house, School of Medicine, Rasht and kept under standard condition in 12 hours light and darks cycle in $21 \pm 3^{\circ} \mathrm{c}$ with full access to adequate water and food. They were treated according to approved principles of vice chancellor of technology and research guidance. Atorvastatin powder was donated from Sobhan Darou pharmaceutical Company $-10 \mathrm{mg} / \mathrm{kg}$ for treatment group. Measured powder (based on weight of each rat) was dissolved in $0.5 \mathrm{cc}$ normal saline. Each rat received an injection once 6 hours after ischemia-reperfusion and 24 later during 3 days intraperitoneally [21].

\section{Experimental groups}

Rats were divided into four equal groups (6 rats in each):

Control: Animals anesthetized with ketamine solution $(100 \mathrm{mg} / \mathrm{kg})$ and Xylazin $(10 \mathrm{mg} / \mathrm{kg})$ [22], and underwent surgical stress in anterior neck with no common carotid clamp.

Ischemia: reperfusion occurred after anesthesia and clamping of common carotid bilaterally for 20 minutes.

Treatment: 6 hours after anesthesia and ischemia-reperfusion the first dose of atorvastatin $(10 \mathrm{mg} / \mathrm{kg})$ administered and next doses 24 hours afterward for 3 days intraperitoneally [21].

Vehicle: After anesthesia and ischemia-reperfusion, normal saline (as solvent of atorvastatin) was injected intraperitoneally. 


\section{Surgical procedure}

Rats anesthetized by ketamine $(100 \mathrm{mg} / \mathrm{kg})$ and Xylazin $(10 \mathrm{mg} / \mathrm{kg})$ intrapreritoneally and their both common carotid arteries and Rt. Common carotid vein were exposed after a $2 \mathrm{~cm}$ incision in anterior neck under sterile condition. In order to prevent stress or damage during clamping, vagus nerve was recognized and dissected carefully. After ligation of common carotid vein's distal end, $2 \mathrm{ml}$ heparinized saline $(100 \mathrm{~mL}$ of $0.9 \%$ saline containing $250 \mathrm{U}$ heparin) was injected and Then $30 \%$ of total blood volume was extracted from the vein both common carotid clamped by Yashargil microclamp for 20min combined with hypotension and then in order to induce reperfusion and establishing natural blood flow in cerebral arteries, clamping was removed, the extracted blood was reinfused and the incision was sutured. Rats were observed until being stable and conscious and were kept is isolated cages for 96 hours. They were anesthetized and their brain was removed by craniotomy and kept in 4\% paraformaldehyde for fixation. After three days and being certain of tissue fixation, they were processed. To investigate cellular death rate Nissl staining was recruited.

\section{Nissl staining}

This method is used for staining Nissl bodies in the cytoplasm's of neurons which are seen in blue-violet color. It is used normally for distinguishing basic structures of healthy neurons from damaged neurons in the brain and spinal cord [23]. After sample fixation and preparation, coronal section with the thickness of $10 \mu$ 2/3-5 mm from posterior Bregma were prepared using a rotary microtome, transported to gelatinized slides and dyed. They were investigated under a light microscope with 400x magnification and only pyramidal neurons with distinct nucleus and nucleoli are considered as healthy living cells. In order to study the whole region of CA1 hippocampus, 3 consecutive photomicrographs were prepared for each sample and pyramidal cells in this region were counted by image tools software and their mean number was measured.

\section{TUNEL staining}

After sample fixation and preparation, by rotary microtom, coronal sections with $3 \mu$ thickness $2-5 \mathrm{~mm}$ from posterior Bregma were obtained. To recognize apoptotic cells, TUNEL kit (Roche Company, Germany) was used for dying according to the manufacturer's protocol. Briefly sections deparaffinized in xylene and hydrated by decreasing gradients of ethanol gradient, then washed in phosphate-buffered saline (PBS) and permeabilized by proteinase $\mathrm{K}(20 \mu \mathrm{g} / \mathrm{ml})$ for 30 minutes at room temperature. Sections were incubated with $3 \% \mathrm{H} 2 \mathrm{O} 2$ in methanol for 10 minutes to block endogenous peroxidase (POD). Then each section incubated in the TUNEL reaction mixture at $37^{\circ} \mathrm{C}$ for $60 \mathrm{~min}$, rinsed with PBS and visualized by using converter-POD for 30 min at $37^{\circ} \mathrm{C}$ with a humidified atmosphere. Sections were rinsed with PBS then 50- 100 $\mu 1 \mathrm{DAB}$ substrate [diaminobenzidine (DAB)] was added and rinsed with PBS. All slides were mounted by a cover slip and observed by a light microscope at $\times 400$ magnification for detecting TUNEL-positive cells.

\section{Statistical Analysis}

After entering data in SPSS version 20, the normality of data distribution was investigated using Shapiro-wilk test. The result was calculated and reported as mean and standard deviation utilizing one-way variance test analysis. If the difference was significant, pair comparison of groups conducted using Tukey-test. P value $<0.05$ was considered significant.

\section{Results}

Regarding to number of viable and degenerated pyramidal CA1 cells, Nissl staining revealed a significant difference between treatment and control groups $(\mathrm{P}<0.05)$ after common right and left carotid clamping for $20 \mathrm{~min}$. The mean number of intact pyramidal cells in CA1 hippocampus in control group was $108.5 \pm 14.2$, in ischemia group $108.5 \pm 10.9$, in vehicle $111.7 \pm 10.3$ and in treatment group was $184.7 \pm 13$ (Figure-1).

Rats treated with atorvastatin showed less cell death and more cell density than ischemic group. The least number of degenerated cells was found in treatment group (receiving Ator- 
vastatin $10 \mathrm{mg} / \mathrm{kg}$ ) after control group. Data analysis showed no significant difference or reduction in number of viable pyramidal cells of CA1 region in treatment group and control $(\mathrm{P}>0.05)$, while the difference was significant in ischemic group compared to control $(\mathrm{P}<0.05)$. The difference between vehicle and control group was significant, indicating that matter containing $0.9 \%$ saline acted as a solvent for atorvastatin has no protective effect on cells in CA1 hippocampus region. Comparison of ischemia and vehicle group revealed a significant difference $(\mathrm{P}<0.05)$. The mean number of viable cells of this region in treatment group was more than other groups except control (Figure-1).

Tissue section comparison in ischemic and vehicle group revealed an increase in number of damaged cells in CA1 region while few of them were found in treatment and control group (Figure-2). Moreover, our findings showed a significant difference in the number of healthy pyramidal cells between ischemic and vehicle groups $(\mathrm{P}<0.05)$.

TUNEL staining revealed that after ischemia-reperfusion the number of apoptotic bodies in CA1 region was different from control group $(\mathrm{P}<0.05)$. The mean of apoptotic bodies in control group was 1 and after inducing ischemia it raised to 13 and in drug receiving group the mean reduced, indicated a

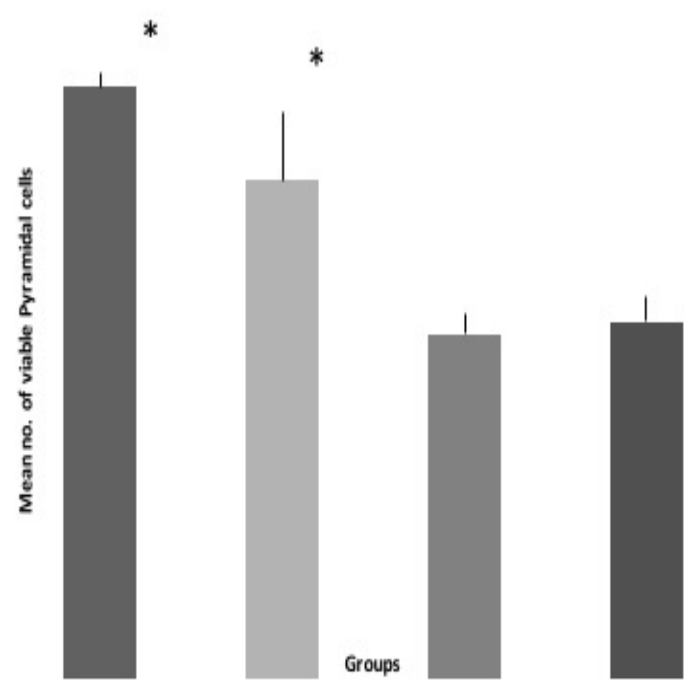

Figure 1. Effect of the Atorvastatin on viability of CA1 Pyramidal cells. Mean no. of viable neurons have decreased in ischemia and vehicle groups significantly compared with those in the control and treatment (atorvastatin) groups. ( ${ }^{*} P<0.05$ compared with control group)

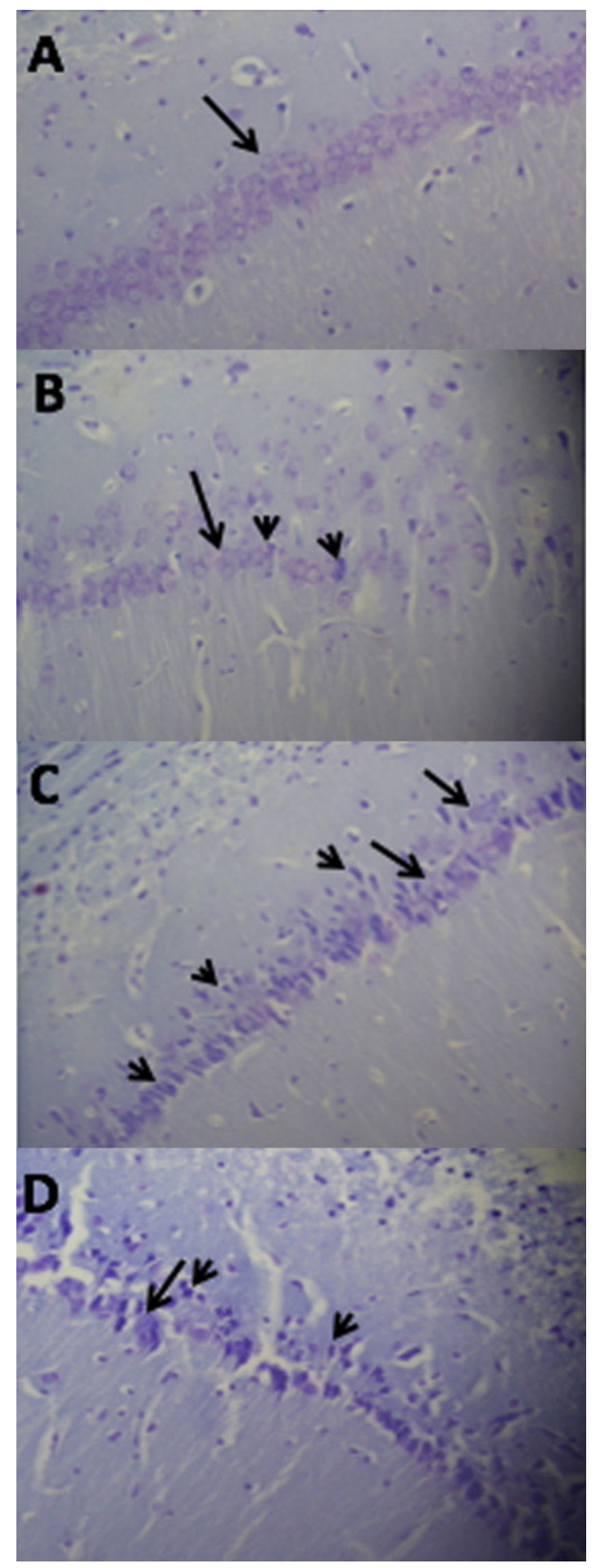

Figure 2. Photomicrograph of the different groups (A: control, B: treatment (atorvastatin), C: vehicle, $D$ : ischemia) showing the degenerated neurons (short arrows) and viable neurons (long arrows) in the CA1 region of hippocampus following Nissl staining. Magnification $\times 400$ 
significant statistical difference $(\mathrm{P}<0.001)$ but no difference between control and treatment group $(\mathrm{P}=0.510)$ nor between ischemia and vehicle group $(\mathrm{P}=0.998)$ (Figure-3).

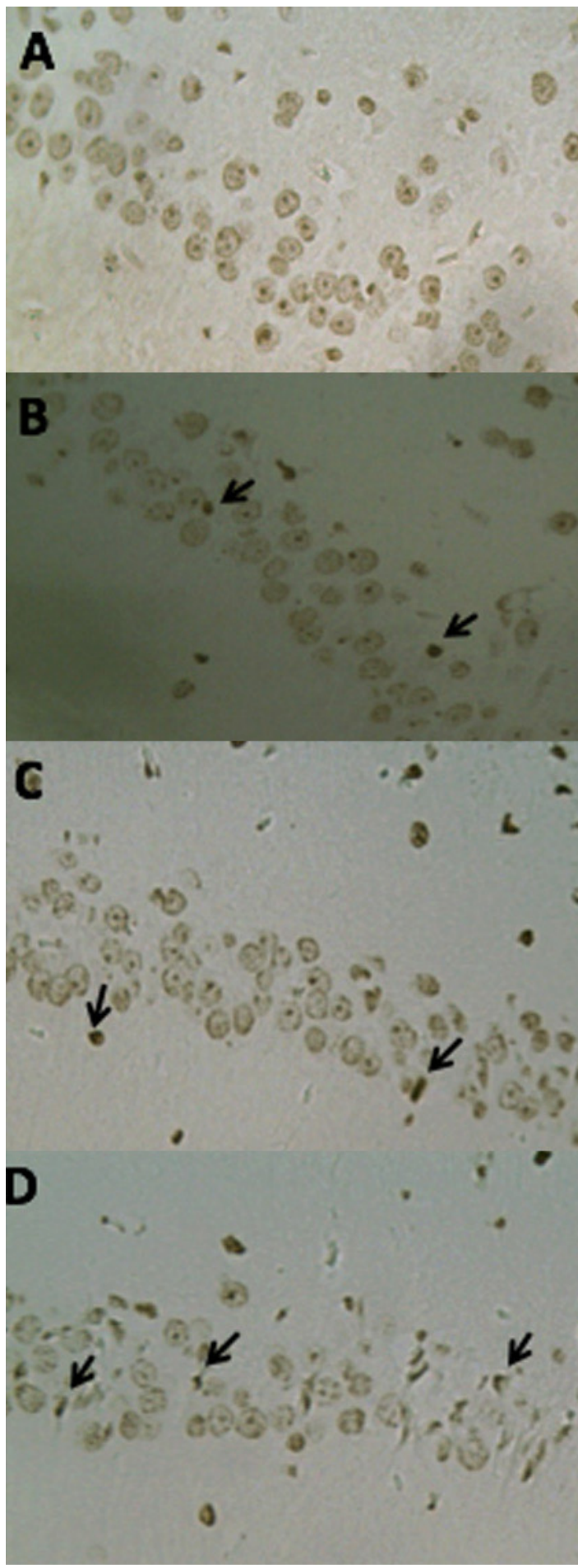

Figure 3. Photomicrograph of the different groups (A: control, B: treatment, C: vehicle, D: ischemia) showing the apoptotic bodies (arrows) in the CA1 region of hippocampus following TUNEL staining. Magnification $\times 400$

\section{Discussion}

The finding of this study indicated that administering Atorvastatin $10 \mathrm{mg} / \mathrm{kg}$ increased healthy pyramidal neural cells and significantly decreased apoptotic bodies in hippocampus CA1 region after transient generalized ischemia. According to conducted studies, the safety of high doses of atorvastatin is verified [24]. In line with the present study findings, other investigations have shown that one weak treatment with Atorvastatin reduces neural damages significantly and increases survival rate and synaptic reconstruction in hippocampus after traumatic cerebral lesions [25]. Following generalized transient ischemia, reactivating oxygenated free radicals like hydrogen peroxide and hydroxyl $(\mathrm{OH})$ are generated which invade targeted tissue and causes a damage more than ischemia alone [10].

Reactive oxygen species (ROS) during neural cell death are produced under different chemical conditions, which play an important role after ischemia and returning of blood stream and cause some reactions of ROS precursors [26]. Researchers have found that eliminating ROS will reduce cerebral ischemic damages [27].Besides reducing cholesterol level, atorvastatin has anti-oxidative and anti-inflammatory properties [18] which are very effective factors in ischemic lesions. This drug eliminates reactivating oxygenating radicals whereby reduces ischemic lesions and resultant cell death. These findings are consistent with our results.

On the other way, primary observation shows that ischemic lesions are generated due to changes of simple biochemical and physiological bindings such as acidosis originating from anaerobic lactate and lack of blood reperfusion due to astrocytes inflammation and pressure to cerebral arteries following blood stream disorders [29].

Similar to our findings, Elevaetal (2009) found that atorvastatin acts as a preservative for arteries after probative cerebral ischemia and reduces hemorrhage after acute stroke [31].

This finding is also consistent with our results in the present study. We believe that atorvastatin with some effect on artery and revascu- 
larization processincreases blood reperfusion in ischemic region and reduces lesions acuity and resultant cell death following ischemia. Studies have indicated that estradiol is neurotrophic and increases neuron survival which can reduces lesions created by cerebral ischemia in gender [32]. Therefore, in order to prevent the effect of preservative effect of female hormone, male wistar rats are used here. The results of the present study indicated that due to anti-oxidative properties, Atorvastatin prevents inflammation and lesions resulting from oxidative induction and also reduces cell death after cerebral ischemia. Another mechanism indicating positive effect of this drug related to prevention of cell death proved in this study is that, Atorvastatin has vasodilatory and preservative effects on arteries which regulate NO synthesis increase in endothelial cell promoting capillary blood stream in ischemic region [20].

Regarding to anti-apoptotic property of Atorvastatin, investigators have concluded that preventive treatment of this drug will inhibit apoptosis processes linked to caspase and will reduce apoptotic neurons number [33]. It seems that increase of the drug administration will increase its preservative effect than low or moderate dose [34]. Furthermore, in most studies $10 \mathrm{mg} / \mathrm{kg}$ dose has been stated to have the most neuroprotective and anti-apoptotic effect [34-37].

\section{Conclusion}

Since our findings showed that $10 \mathrm{mg} / \mathrm{kg}$ atorvastatin can prevent apoptosis and cell death in pyramidal hippocampus CA1 neurons after ischemia-reperfusion and also reduces cerebral ischemic lesions, to testify this results, investigation on other sensitive ischemic areas is recommended.

\section{Acknowledgement}

We would like to express our sincere thanks toward; Sobhan Darou Pharmaceutical Company, Rasht; Molecular and Cellular Research Center, Islamic Azad University Tehran Medical Sciences Branch; Embryology and Histology Laboratory, Medical School, Rasht; and Mr. Moravati in charge of Animal House in Medical School of Rasht.

\section{Conflict of interest}

None declared.

\section{References}

1. Bokura H, Robinson RG. Long term cognitive impairment associated with caudate stroke. Stroke 1997; 28:970-975.

2. Braduik B, Sonesson B, Holtas S. Spatial impairment hallowing right hemisphere transient ischemic attackts in patients without carotid artery stenosis. Acta Neural Scand 1989; 80: 411-8.

3. Godefroy O, Rousseaux M, Pruro JP, Cabara M, Leys D. Neuropsychological changes related to unilateral lenticostriate infarcts. J Neurol Neurosurg Psychiatry 1994; 57(4):480-5

4. Petito CK, Feldmann E, Pulsinelli WA, Plum F. Delayed hippocampal damage in humans following cardiorespiratory arrest. Neurology 1987; 37: 1281-6.

5. Kesner RP, Adelstein T, Crutcher K. Equivalent spatial location memory deficits in rats with medial septum or hippocampal formation lesions and patients with dementia of the Alzheimer's type. Brain Cogn 1989;9(2):289300.

6. Alipanahzade H, Soleimani M, SoleimaniAsl S, Mehdizadeh M, Katebi M. Effect of transforming growth factor alpha of dentytejyrus neurons and pyramidal cells of CA1 subfiled of hippocampus following ischemia- reperfusion in Rats.J Gorgan Uni Med Sci 2012, 14(3): 26-32.

7. Deckert J, Jorgensen MB. Evidence for pre- and postsynaptic localization of adenosine A1 receptors in the CA1 region of rat hippocampus: a quantitative autoradiographic study. Brain Res 1988; 446(1): 161-4.

8. Zhang J, Piantadosi CA. Prolonged production of hydroxyl radical in rat hippocampus after brain ischemia-reperfusion is decreased 
by 21-aminosteroids. Neurosci Lett. 1994; 177(1-2):127-30.

9. Simonová Z, Sterbová K, Brozek G, Komárek V, Syková E. Postnatal hypobaric hypoxiain rats impairs water maze learning and morphology of neurones and macroglia in cortex and hippocampus. Behav Brain Res 2003; 141: 195-205.

10. Zini I, Tomasi A, Grimaldi R, Vannini V, Agnati LF. Detection of free radicals during brain ischemia and reperfusion by spin trapping and microdialysis. Neurosci Lett.1992; 138(2): 279-82.

11. Pulsinelli WA, Brierley JB, Plum F. Temporal profile of neuronal damage in a model of transient forebrain ischemia. Ann Neurol 1982;11:491-8.

12. The National Institute of Neurological Disorders and Stroke rt-PA Stroke Study Group. Tissue plasminogen activator for acute ischemic stroke. N Engl J Med 1995; 333:1581-7.

13. Graaf MR. The risk of cancer in users of statins. J Clin Oncol. 2004; 22(12): 2388-94

14. Zacco A, Togo J, Spence K, Ellis A, Lloyd D, Furlong S, et al. 3-Hydroxy-3-methylglutaryl coenzyme A reductase inhibitors protect cortical neurons from excitotoxicity. J. Neurosci 2003; 23:11104-11.

15. Tanaka N, Katayama Y, Katsumata T, Otori T, Nishiyama Y. Effects of long-term administration of HMG-CoA reductase inhibitor, atorvastatin, on stroke events and local cerebral blood flow in stroke-prone spontaneously hypertensive rats. Brain Res 2007; 1169: 125-32

16. Endres M, Laufs U, Huang Z, Nakamura T, Huang P, Moskowitz MA, et al. Stroke protection by 3-hydroxy-3-methylglutaryl (HMG)-CoA reductase inhibitors mediated by endothelial nitric oxide synthase. Proc Natl Acad Sci U S A 1998; 95: 8880-5.

17. Schachter M. Chemical, pharmacokinetic and pharmacodynamic properties of statins: an update. Fundam Clin Pharmacol 2005; 19(1): 117-25.

18. Kawai H, Deguchi SH, Deguchi K, Yamashita T, Ohta Y, Omote Y, et al. Protection against ischemic stroke damage by synergistic treatment with amlodipine plus atorvastatin in Zucker metabolic rat. Brain Research 2011; 1382: 308-14.

19. Laufs U, Gertz K, Huang P, Nickenig G, Bohm M, Dirnagl U, et al. Atorvastatin upregulates type iii nitric oxide synthase in thrombocytes, decreases platelet activa- tion, and protects from cerebral ischemia in normocholesterolemic mice. Stroke 2000; 31: 2442-9.

20. Clarke RM, Lyons A, O’Connell F, Deighan BF, Barry CE, Anyakoha NG, et al. A pivotal role for interleukin-4 in atorvastatin-associated neuroprotection in rat brain. J Biol Chem 2008; 283: 1808-17.

21. Céspedes-Rubio A, JuradoHYPERLINK "http://www.ncbi.nlm.nih.gov/pubmed?ter$\mathrm{m}=$ Jurado\%20FW\%5BAuthor\%5D\&cauthor=true\&cauthor_uid=20936696" FW, Cardona-Gómez GP. p1 20 catenin/ $\alpha \mathrm{N}$ - catenin are molecular targets in the neuroprotection and neuronal plasticity mediated by atorvastatin after focal cerebral ischemia. J Neurosci Res. 2010; 88(16):3621-34

22. Zamani M, Rasooli H, Mehdizadeh M, Nobakht M, Zamani F, Soleimani M. Protective effect of Adenosine A1 receptor and ascorbic acid on hippocampal neuronal density and memory disorder in ischemia reperfusion induced Rats. J Gorgan Uni Med Sci. 2013; 14(4):52-9.

23. Kiernan JA. Histological, histochemical methods. 3rd Edition. Oxford: Butterworth Heinemann; 1999.

24. Waters DD. Safety of high-dose atorvastatin therapy. Am J Cardiol 2005; 96 (5A): $69 \mathrm{~F}-75 \mathrm{~F}$

25. Lu D, Goussev A, Chen J, Pannu P, Li Y, Mahmood A, Chopp M. Atorvastatin reduces neurological deficit and increases synaptogenesis, angiogenesis, and neuronal survival in rats subjected to traumatic brain injury. $\mathrm{J}$ Neurotrauma 2004; 21(1): 21-32.

26. Chan PH. Role of oxidants in ischemic brain damage. Stroke 1996; 27: 1124-1129.

27. Lewen A, Matz P, Chan PH. Free radical pathways in CNS injury. J Neurotrauma 2000 17: 871-90.

28. Wityk RJ, Goldsborough MA, Hillis A, Beauchamp N, Barker PB, Borowicz LM Jr, et al. Diffusion and perfusion-weighted brain magnetic resonance imaging in patients with neurologic complications after cardiac surgery. Arch Neurol 2001; 58: 571-6.

29. Ruiz V. Statins upregulate CD 36 expressin in human monocytes an affect strengthened when combined with PPAR-gamma ligand putative contribution of Rho GTPase in statin-induced CD36 expression. Biochem Pharmacol. 2004; 67(2): 303-13.

30. Gertz K, Laufs U, Lindauer U, Nickenig G, Böhm M, Dirnagl U, et al. Withdrawal of statin treatment abrogates stroke protection in 
mice. Stroke 2003; 34: 551-7.

31. Elewa HF, KozakHYPERLINK "http:// www.ncbi.nlm.nih.gov/pubmed?ter$\mathrm{m}=$ Kozak\%20A\%5BAuthor\%5D\&cauthor=true\&cauthor uid=19478137" A, El-HYPERLINK “"http://www.ncbi.nlm. nih.gov/pubmed?term=El-Remessy $\% 20$ AB\%5BAuthor\%5D\&cauthor=true\&cauthor_uid=19478137'RemessyHYPERLINK "http://www.ncbi.nlm.nih.gov/pubmed?ter$\mathrm{m}=$ El-Remessy\%20AB\%5BAuthor\%5D\&cauthor=true\&cauthor_uid=19478137" AB, Frye RF, Johnson MH, ErgulHYPERLINK "http://www.ncbi.nlm.nih.gov/pubmed?ter$\mathrm{m}=$ Ergul $\% 20 \mathrm{~A} \% 5 \mathrm{BAuthor} \% 5 \mathrm{D} \& \mathrm{cau}-$ thor=true\&cauthor_uid=19478137" A, et al. Early atorvastatin reduces hemorrhage after acute cerebral ischemia in diabetic rats. JHYPERLINK "http://www.ncbi. nlm.nih.gov/pubmed/?term=early+atorvastatin+reduces+hemorrhage+after" HYPERLINK "http://www.ncbi.nlm.nih. gov/pubmed/?term=early+atorvastatin +reduces +hemorrhage+after"Pharmacol Exp Ther 2009; 330(2): 532-540.

32. Weaver CE HYPERLINK "http://www.ncbi. nlm.nih.gov/pubmed/?term $=$ Weaver $\% 20$ CE\%20Jr\%5BAuthor\%5D\&cauthor=true\&cauthor_uid=9252035'Jr, Park-Chung M, Gibbs TT, FarbHYPERLINK "http://www. ncbi.nlm.nih.gov/pubmed/?term=Farb $\% 20$ DH\%5BAuthor\%5D\&cauthor=true\&cauthor_uid=9252035" DH. 17beta-Estradiol protects against NMDA-induced excitotoxicity by direct inhibition of NMDA receptors. Brain Res. 1997 Jul 4;761(2):338-41.

33. Cheng G, Wei L, ZhiHYPERLINK "http:// www.ncbi.nlm.nih.gov/pubmed/?ter$\mathrm{m}=$ Zhi-Dan\%20S\%5BAuthor\%5D\&cauthor=true\&cauthor_uid=19159448"-Dan S, Shi-HYPERLINK "http://www.ncbi.nlm. nih.gov/pubmed/?term=Shi-Guang $\% 20$ Z\%5BAuthor\%5D\&cauthor=true\&cauthor uid=19159448"GuangHYPERLINK "http://www.ncbi.nlm.nih.gov/pubmed/?ter$\mathrm{m}=$ Shi-Guang\%20Z\%5BAuthor\%5D\&cauthor=true\&cauthor_uid=19159448" Z, Xiang-Zhen HYPERLINK "http://www.ncbi. nlm.nih.gov/pubmed/?term=Xiang-Zhen $\% 20$ L\%5BAuthor\%5D\&cauthor=true\&cauthor uid=19159448"L. Atorvastatin ameliorates cerebral vasospasm and early brain injury after subarachnoid hemorrhage and inhibits caspase-dependent apoptosis pathway. BMC HYPERLINK "http://www.ncbi.nlm. nih.gov/pubmed/19159448”NeurosciHYPERLINK “http://www.ncbi.nlm.nih.gov/ pubmed/19159448”. 2009;10:7.

34. DoustarY, Mohajeri D. The anti-apoptotic effects of atorvastatin in isoproterenol induced experimental heart failure. ZJRMS 2011, 13(2): 13-9.

35. Laxman D, Zeng H. Atorvastatin inhibits Fas expression in ischemia-reperfusion induced myocardial cell injury. JRMS 2006; 11(3): 137-45.

36. Kawai H1, DeguchiHYPERLINK "http:// www.ncbi.nlm.nih.gov/pubmed/?term=Deguchi\%20S\%5BAuthor\%5D\&cauthor=true\&cauthor_uid=21276424" S, DeguchiHYPERLINK "http://www.ncbi. nlm.nih.gov/pubmed/?term=Deguchi $\% 20$ K\%5BAuthor\%5D\&cauthor=true\&cauthor_uid=21276424” K, Yamashita T, OhtaHYPERLINK "http://www.ncbi. nlm.nih.gov/pubmed/?term=Ohta $\% 20$ Y\%5BAuthor\%5D\&cauthor=true\&cauthor_uid=21276424" Y, OmoteHYPERLINK "http://www.ncbi.nlm.nih.gov/pubmed/?ter$\mathrm{m}=$ Omote\%20Y\%5BAuthor\%5D\&cauthor=true\&cauthor_uid=21276424"Y, et al. Protection against ischemic stroke damage by synergistic treatment with amlodipine plus atorvastatin in Zucker metabolic rat. Brain Res. 2011; 1382:308-14.

37. CéspedesHYPERLINK "http://www.ncbi. nlm.nih.gov/pubmed/?term $=\mathrm{C} \% \mathrm{C} 3 \% \mathrm{~A} 9 \mathrm{~s}$ pedes-Rubio $\% 20 \mathrm{~A} \% 5 \mathrm{BAuthor} \% 5 \mathrm{D} \& \mathrm{cau}-$ thor=true\&cauthor uid=20936696"-Rubio A1, JuradoHYPERLINK "http://www.ncbi. nlm.nih.gov/pubmed/?term=Jurado $\% 20$ FW\%5BAuthor\%5D\&cauthor=true\&cauthor_uid=20936696" FW, Cardona-Gómez GP. p120 catenin/ $\alpha \mathrm{N}$-catenin are molecular targets in the neuroprotection and neuronal plasticity mediated by atorvastatin after focal cerebral ischemia. J HYPERLINK "http://www.ncbi.nlm.nih. gov/pubmed/20936696”NeurosciHYPERLINK “http://www.ncbi.nlm.nih.gov/ pubmed/20936696” Res. 2010; 88(16):362134. 